\title{
Preoperative Cognitive Impairment As a Predictor of Postoperative Outcomes in a Collaborative Care Model
}

\author{
Kabli Zietlow, MD, * Shelley R. McDonald, DO, PhD, * Richard Sloane, MPH, Jeffrey Browndyke, \\ $\mathrm{PhD},{ }^{*}$ Sandhya Lagoo-Deenadayalan, MD, PhD, ${ }^{\xi}$ and Mitchell T. Heflin, MD, MHS*
}

OBJECTIVES: To compare postoperative outcomes of individuals with and without cognitive impairment enrolled in the Perioperative Optimization of Senior Health (POSH) program at Duke University, a comanagement model involving surgery, anesthesia, and geriatrics.

DESIGN: Retrospective analysis of individuals enrolled in a quality improvement program.

SETTING: Tertiary academic center.

PARTICIPANTS: Older adults undergoing surgery and referred to POSH $(\mathrm{N}=157)$.

MEASUREMENTS: Cognitive impairment was defined as a score less than 25 out of 30 (adjusted for education) on the St. Louis University Mental Status (SLUMS) Examination. Median length of stay (LOS), mean number of postoperative complications, rates of postoperative delirium (POD, \%), 30-day readmissions (\%), and discharge to home $(\%)$ were compared using bivariate analysis.

RESULTS: Seventy percent of participants met criteria for cognitive impairment (mean SLUMS score 20.3 for those with cognitive impairment and 27.7 for those without). Participants with and without cognitive impairment did not significantly differ in demographic characteristics, number of medications (including anticholinergics and benzodiazepines), or burden of comorbidities. Participants with and without cognitive impairment had similar LOS $(P=.99)$, cumulative number of complications $(P=.70)$, and 30-day readmission $(P=.20)$. POD was more common in those with cognitive impairment ( $31 \%$ vs $24 \%$ ), but the difference was not significant $(P=.34)$. Participants without cognitive impairment had higher rates of discharge to home $(80.4 \%$ vs $65.1 \%, P=.05)$.

CONCLUSION: Older adults with and without cognitive impairment referred to the $\mathrm{POSH}$ program fared similarly on most postoperative outcomes. Individuals with

From the *Division of Geriatrics, Department of Internal Medicine; ${ }^{\dagger}$ Center for Aging; ${ }^{*}$ Division of Geriatric Behavioral Health, Department of Psychiatry; and ${ }^{\S}$ Department of Surgery, Duke University Health Systems, Durham, North Carolina.

Address correspondence to Kahli Zietlow, GRECC (182) VAMC, 508 Fulton Street, Durham, NC 27705. E-mail: kahli.zietlow@duke.edu

DOI: $10.1111 /$ jgs. 15261 cognitive impairment may benefit from perioperative geriatric comanagement. Questions remain regarding the validity of available measures of cognition in the preoperative period. J Am Geriatr Soc 2018.

Key words: cognitive impairment; older adults; postoperative outcomes; co-management

A pproximately one-third of elective surgical procedures $A$ are performed on adults aged 65 and older, and this population has a high burden of postsurgical morbidity and mortality. ${ }^{1}$ As the population ages, an increasing number of surgeries will be performed on these higher-risk older adults. ${ }^{2}$ Traditional preoperative risk assessment tools, such as the American Society of Anesthesiologists (ASA) Physical Classification System, focus on medical factors associated with risk of adverse postoperative outcomes, ${ }^{3}$ but such tools do not account for risk factors unique to older adults such as malnourishment, impaired mobility, cognitive impairment, and frailty. Geriatric-specific risk stratification models are better able to predict outcomes in older adults. ${ }^{1,4}$ A growing literature supports incorporation of tools used for geriatric assessment as part of the preoperative evaluation of older adults undergoing elective surgery. ${ }^{5-7}$ The Perioperative Optimization of Senior Health (POSH) program is an innovative care model developed at Duke University that aims not only to assess surgical risk in older adults accurately, but also to improve clinical outcomes through early multidisciplinary risk assessment and targeted interventions throughout the perioperative period. ${ }^{8}$

Cognitive ability is one of the most important perioperative risk indicators, and the prevalence of mild cognitive impairment (MCI) and dementia are expected to rise. ${ }^{9}$ Preoperative cognitive impairment, whether MCI or dementia, has been linked to higher rates of postoperative delirium (POD), ${ }^{10,11}$ which, in turn, has been associated with greater morbidity and long-term declines in functional status and cognition. ${ }^{12,13}$ Despite these findings, the literature 
exploring whether cognitive impairment itself is an independent risk factor for other postoperative complications is scarce and inconsistent, and individuals with cognitive impairment are often excluded from clinical trials. ${ }^{14-18}$ The aim of our study was to compare postoperative outcomes in individuals enrolled in the POSH program with and without cognitive impairment as assessed using the St. Louis University Mental Status (SLUMS) Examination. ${ }^{19}$ As a secondary aim, we explored whether performance on SLUMS was predictive of POD.

\section{METHODS}

\section{POSH Quality Improvement Program Description}

The Duke University POSH program is a quality improvement initiative that began in 2011. Individuals referred to the POSH program undergo multidisciplinary preoperative assessment. At their preoperative appointment, a nurse, social worker, geriatrician, and anesthesia advanced practice provider evaluate them. The team collaborates to collect relevant demographic and medical data. In addition to a comprehensive physical examination, cognition is screened using the SLUMS, which a trained provider administers to ensure standardization of scoring. A plan is developed to provide medical optimization before surgery, with particular attention to factors relevant to older adults. Examples of things addressed include establishing advance directives, ensuring that vision and hearing aids are available in the hospital, optimizing nutritional status, and discontinuing inappropriate medications.

Postoperatively, individuals are admitted to surgical services with collaborative care from the geriatrics consultation team, which assists with delirium prevention and care, management of medical comorbidities, pain control, promotion of mobility, and minimization of polypharmacy. The internal review board at Duke reviewed the POSH service description and planned analyses and determined them to be exempt as a quality improvement project.

\section{Participants}

POSH program participants evaluated between July 2014 and June 2015 were eligible for this analysis. Individuals undergoing elective inpatient surgery were referred to POSH when the decision was made to proceed with a surgical intervention. Referral was at the discretion of their surgeons. Suggested eligibility criteria for referral included age 85 and older or aged 65 to 84 and meeting at least one qualifying criterion: preexisting diagnosis of MCI or dementia, poor nutritional status $(\geq 10$ pounds unintentional weight loss in preceding 12 months or body mass index $\leq 23.0 \mathrm{~kg} / \mathrm{m}^{2}$ ), visual impairment (binocular vision $\leq 20 / 70$ ), multimorbidity ( $\geq 2$ chronic medical conditions), and polypharmacy ( $\geq 5$ prescription medications).

\section{Measures}

Presence or absence of cognitive impairment was assessed using SLUMS, which is a 30-point, 11-item cognitive screening tool developed to detect possible cognitive decline. ${ }^{9}$ Thresholds for SLUMS scores suggestive of cognitive impairment are adjusted for education level. In individuals with at least a high school level of education, a score of 27 or higher suggests grossly intact cognition, a score of 21 to 26 suggests MCI, and a score less than 21 suggests more-severe deficits that may be reflective of dementia. These SLUMS total score thresholds are adjusted for individuals with less than a high school education (20-24 suggestive of MCI; $\leq 19$ suggestive of dementia). We created a dichotomous sample of participants with and without cognitive impairment based on their SLUMS performance and education level (cognitive impairment defined as $<27$ for individuals with a high school level of education, $<25$ for individuals without a high school education).

We compared baseline characteristics of POSH participants with and without cognitive impairment, including demographic data, smoking status, alcohol intake, use of vision and hearing aids, and presence of comorbidities associated with cognitive impairment, including anxiety, depression, diabetes, obesity, hypertension, prior stroke or transient ischemic attack, and coronary artery disease. ${ }^{20}$ Comorbidities were ascertained from review of medical records and self-report. We also compared Cumulative Index Rating Scale scores, which is a severity score for degree of illness in 13 body systems, with higher scores indicating overall higher burden of illness. ${ }^{17,21}$ We compared baseline number of medications, use of benzodiazepines, and anticholinergic burden as assessed using the Anticholinergic Cognitive Burden Scale. ${ }^{22}$ Finally, we determined baseline number of deficiencies in activities of daily living (ADLs) and instrumental activities of daily living (IADLs) as participants or accompanying family members reported. Information from a preoperative POSH assessment was entered and managed using Research Electronic Data Capture (REDCap), a secure, web-based application that provides audit trails for data tracking and export procedures. $^{23}$

Outcomes data was assessed using individual-level institutional data from clinical, operational, administrative, and billing systems. Medical record numbers were used to obtain information on length of stay (LOS), readmissions, and discharge destination. All 7- and 30-day readmission data were verified using chart review. The inpatient geriatrics consultation team diagnosed POD using the Confusion Assessment Method (CAM) criteria or the CAM-ICU, if appropriate. ${ }^{24}$ The geriatrics consultation team performed CAM assessments daily. Postoperative complications were identified according to International Classification of Diseases, Ninth Revision, Clinical Modification codes associated with the index surgical hospitalization obtained from billing data. Bivariate analyses were conducted to determine whether cognitive impairment was associated with LOS, all-cause 7- or 30-day readmission rates, discharge to home, incidence of POD, and a composite outcome of common postoperative medical complications (other than POD) that occurred during the course of hospitalization. Finally, we assessed the predictive ability of SLUMS to assess POD risk using receiver operating characteristic (ROC) curves. 


\section{Statistical Analysis}

The main analytical aim of this study was to describe and compare characteristics, performance measures, and clinical outcomes of POSH participants undergoing surgery according to cognitive status category based on SLUMS score. Bivariate analyses of cognitive impairment groups were conducted using the chi-square test for categorical variables and the parametric t-test for continuous variables (if normally distributed) or the nonparametric Wilcoxon rank-sum test (if not normally distributed). A secondary aim was to explore the most informative SLUMS cut-point in this sample to inform the likelihood of POD. ROC analysis was used to determine the SLUMS score with the optimal cut-point, primarily guided by inspecting relative c-statistic values. All analyses were conducted using SAS version 9.4 (SAS Institute, Inc., Cary, NC).

\section{RESULTS}

\section{Participants}

One hundred fifty-seven individuals were included in this analysis. The majority resided at home before surgery $(98.8 \%)$. Seventy-three $(46.5 \%)$ underwent neurosurgical procedures, $69(43.9 \%)$ general (gastrointestinal, hepatopancreatobiliary, colorectal, vascular), $12(7.6 \%)$ urological, $1(0.6 \%)$ orthopedic, $1(0.6 \%)$ cardiac, and 1 $(0.6 \%)$ thoracic. At Duke University Health Systems, 19,366 urological surgeries, 2,191 general surgeries, and 634 spinal neurosurgeries were performed electively on adults aged 65 and older during the study period, meaning that $0.06 \%, 3.1 \%$, and $11.5 \%$ of individuals from each service, respectively, were referred to POSH. There was no difference in distribution of surgical services of participants with and without cognitive impairment. Frequency of laparoscopic and open procedures on the general surgery service was similar between participants with and without cognitive impairment $(21.7 \%$ and $23.5 \%$ laparoscopic procedures respectively, $\mathrm{P}=0.79$ ).

Based on their total SLUMS scores, 106 (67.5\%) participants met criteria for cognitive impairment. Mean SLUMS score was $20.3 \pm 4.8$ in participants with cognitive impairment and $27.7 \pm 2.9$ in those without. The study groups were similar in terms of demographic characteristics, substance use, past medical history, and medication use (Table 1 ). The study groups differed significantly only in functional status; $20.8 \%$ of participants with cognitive impairment and $5.8 \%$ of participants without were dependent in at least $1 \mathrm{ADL}(P=.02)$. The proportion of participants with deficiencies in one or more IADLs did not significantly differ $(47.1 \%$ vs $56.6 \%, P=.26)$. Additionally, a nonsignificant difference in visual impairment was observed between participants with $(90.6 \%)$ and without $(80.4 \%)$ cognitive impairment $(P=.07)$.

\section{Outcomes}

The two populations did not significantly differ in terms of median LOS, or 7- and 30-day readmission rates (Table 2). Participants with cognitive impairment had higher rates of POD ( $31.4 \%$ vs $24.0 \%)$, but this difference

\section{Table 1. Participant Baseline Characteristics}

\begin{tabular}{|c|c|c|c|}
\hline Variable & $\begin{array}{c}\text { No Cognitive } \\
\text { Impairment, } n=51\end{array}$ & $\begin{array}{l}\text { Cognitive Impairment, } \\
\qquad n=106\end{array}$ & P-Value \\
\hline \multicolumn{4}{|l|}{ Demographic data } \\
\hline Age, median (SD) & $73.7(5.8)$ & $75.5(7.5)$ & .11 \\
\hline Male, $n(\%)$ & $23(46.0)$ & $53(52.0)$ & .49 \\
\hline High school graduate, $\mathrm{n}(\%)$ & $45(88.2)$ & $92(86.8)$ & .80 \\
\hline \multicolumn{4}{|l|}{ Medical history } \\
\hline Current or prior smoker, n (\%) & $23(46.0)$ & $58(56.9)$ & .21 \\
\hline Alcohol, $\geq 1$ drinks per week, $n(\%)$ & $11(22.0)$ & $16(15.1)$ & .29 \\
\hline Vision impairment, $\mathrm{n}(\%)$ & $41(80.4)$ & $96(90.6)$ & .07 \\
\hline Hearing impairment, $n(\%)$ & $16(31.4)$ & $46(43.4)$ & .15 \\
\hline Body mass index, $\mathrm{kg} / \mathrm{m}^{2}$ mean & 29.5 & 28.2 & .20 \\
\hline Cumulative IIIness Rating Scale score, mean & 7.8 & 8.7 & .14 \\
\hline Anxiety, n (\%) & $15(29.1)$ & $31(29.25)$ & .98 \\
\hline Depression, n (\%) & $15(29.1)$ & $34(32.1)$ & .74 \\
\hline Diabetes, n (\%) & $12(23.5)$ & 25 (23.6) & .99 \\
\hline Obesity, $\mathrm{n}(\%)$ & $12(23.5)$ & $24(22.6)$ & .90 \\
\hline Hypertension, $\mathrm{n}(\%)$ & $12(23.5)$ & $36(34.0)$ & .18 \\
\hline History of stroke or transient ischemic attack, $\mathrm{n}(\%)$ & $3(5.9)$ & $14(13.2)$ & .17 \\
\hline Coronary artery disease & $15(29.4)$ & $34(32.1)$ & .74 \\
\hline \multicolumn{4}{|l|}{ Pharmacy } \\
\hline Number of medications, mean $\pm S D$ & $12.0 \pm 7.4$ & $12.4 \pm 5.8$ & .70 \\
\hline Anticholinergic burden, mean $\pm \mathrm{SD}^{\mathrm{a}}$ & $1.12 \pm 1.8$ & $1.21 \pm 1.65$ & .75 \\
\hline Benzodiazepine use, n (\%) & $9(17.7)$ & $19(18.1)$ & .94 \\
\hline$\geq 1$ ADL deficiencies, $n(\%)$ & $3(5.6)$ & $22(20.8)$ & .02 \\
\hline$\geq 1$ instrumental ADL deficiencies, $\mathrm{n}(\%)$ & $24(47.1)$ & $60(56.6)$ & .26 \\
\hline
\end{tabular}

${ }^{a}$ Assessed using the Anticholinergic Burden Scale. ${ }^{23}$

$\mathrm{ADL}=$ activity of daily living; $\mathrm{SD}=$ standard deviation. 
Table 2. Outcome Data

\begin{tabular}{lccc}
\hline Use and Complications & $\begin{array}{c}\text { No Cognitive } \\
\text { Impairment, } \\
\mathbf{n}=\mathbf{5 1}\end{array}$ & $\begin{array}{c}\text { Cognitive } \\
\text { Impairment, } \\
\mathbf{n}=\mathbf{1 0 6}\end{array}$ & P-Value \\
\hline $\begin{array}{l}\text { Length of stay, days, } \\
\text { median }\end{array}$ & 4.0 & 4.0 & .99 \\
$\begin{array}{l}\text { 7-day readmissions, } \mathrm{n}(\%) \\
\text { 30-day readmissions, } \mathrm{n}(\%)\end{array}$ & $0(0.0)$ & $3(2.8)$ & .23 \\
$\begin{array}{l}\text { Discharged to home, } \\
\text { n (\%) }\end{array}$ & $41(80.4)$ & $69(65.1)$ & .05 \\
$\begin{array}{l}\text { Postoperative delirium, } \\
\text { n (\%) }\end{array}$ & $12(24.0)$ & $33(31.4)$ & .34 \\
$\begin{array}{l}\text { Number of postoperative } \\
\text { complications, }\end{array}$ & $0.88 \pm 0.55$ & $0.98 \pm 0.65$ & .71 \\
mean \pm standard deviation & & & \\
\hline
\end{tabular}

Table 3. Individual Postoperative Adverse Events Other Than Postoperative Delirium

\begin{tabular}{cc}
$\begin{array}{c}\text { No Cognitive } \\
\text { Impairment, } \\
n=51\end{array}$ & $\begin{array}{c}\text { Cognitive } \\
\text { Impairment, } \\
n=106\end{array}$ \\
\hline
\end{tabular}

Complication

\begin{tabular}{lll}
\hline $\begin{array}{l}\text { Deep vein thrombosis, } \\
\text { pulmonary embolism }\end{array}$ & $1(2.0)$ & $1(0.9)$ \\
Wound infection & $0(0)$ & $2(1.9)$ \\
Wound dehiscence & $0(0)$ & $0(0)$ \\
Urinary tract infection & $0(0)$ & $3(2.8)$ \\
Urinary retention & $2(3.9)$ & $5(4.7)$ \\
Pneumonia & $2(3.9)$ & $2(1.9)$ \\
Sepsis & $1(2.0)$ & $4(3.8)$ \\
Cardiac arrhythmia & $3(5.9)$ & $9(8.5)$ \\
Acute coronary syndrome & $1(2.0)$ & $1(0.9)$ \\
Cardiac arrest & $1(2.0)$ & $2(1.9)$ \\
Acute cerebral & $0(0)$ & $2(1.9)$ \\
vascular accident & $1(2.0)$ & $1(0.9)$ \\
Ileus & $7(13.7)$ & $7(6.6)$ \\
Nausea, vomiting & $5(9.8)$ & $9(8.5)$ \\
Acute kidney injury & $2(3.9)$ & $4(3.8)$ \\
Hemorrhage & $0(0)$ & $0(0)$ \\
Hypoglycemia & $1(2.0)$ & $3(1.9)$ \\
Hyperglycemia & $0(0)$ & $1(0.9)$ \\
Falls & $0(0)$ & $0(0)$ \\
Pressure ulcers & $0(0)$ & $0(0)$ \\
Alcohol withdrawal & &
\end{tabular}

was not statistically significant $(P=.34)$. Rates of individual medical complications, excluding POD, were low and did not differ significantly between the two populations (Table 3). Likewise, a composite outcome of the total number of medical complications was not significantly different between the two groups. Participants with cognitive impairment had a lower rate of discharge to home $(80.4 \%)$ than to other destinations (skilled nursing facility, assisted living facility, or hospice, $65.1 \%, P=.05$ ).

\section{SLUMS As a Predictor of Postoperative Delirium}

ROC curves revealed an equivocal predictive capacity of SLUMS for POD (Figure S1). At the traditional cut-points of 27 and 25 (cut-points for individuals with MCI with and without high school education, respectively), as well as 20 and 19 (cut-points for dementia), the c-statistic was less than 0.60 for all reported values. The optimal predictive value for the SLUMS was a score of $19(0-19$ vs $\geq 20$, c-statistic $=0.54)$.

\section{DISCUSSION}

Available data suggest that preexisting cognitive impairment predicts incident POD, which, in turn, can impair long-term cognitive functioning. ${ }^{10,15,25}$ Preoperative cognitive impairment has also been linked to the incidence of other adverse outcomes, including postoperative complications, longer hospital stay, and functional decline. ${ }^{26-29}$ Nonetheless, data on the association between preoperative cognitive impairment and postoperative outcomes are limited. ${ }^{13,30}$ Furthermore, individuals with cognitive impairment are often excluded from clinical trials. ${ }^{18}$

In this analysis comparing individuals with and without cognitive impairment undergoing elective surgery, individuals with cognitive impairment had higher rates of discharge to a facility than those without cognitive impairment. This is not a surprising outcome, because individuals with cognitive impairment would be less likely to function independently after surgery. Given the low rates of individuals residing in assisted living before surgery $(1.2 \%)$, we do not think that prior home environment meaningfully affected discharge destination.

Participants with cognitive impairment did not have longer hospital stays or higher readmission rates than their cognitively intact counterparts. Likewise, participants with cognitive impairment did not have significantly different rates of POD or other postoperative complications. There are a number of possible explanations for this finding. In our study population, the rate of cognitive impairment was $64 \%$, which is much higher than the rate of cognitive impairment in the general population (estimated to be $20-25 \%$ ). This high rate may represent preferential referrals of individuals with cognitive impairment from participating surgeons. Additionally, the fatigue of multiple provider visits, the stress of upcoming surgery, and the predisposing medical condition necessitating surgery may have contributed to poor performance on cognitive screening, artificially inflating the rates of cognitive impairment in the preoperative evaluation setting. Thus, it is possible that overestimation of rates of cognitive impairment do not reflect clinically meaningful disease and hence the similar outcomes in individuals with and without cognitive impairment in our study population.

A second explanation is that participants may have benefited from the geriatric comanagement model, mitigating the effect of cognitive impairment on postoperative adverse events. Using a multidisciplinary approach, POSH providers address and optimize geriatric syndromes, such as malnutrition and polypharmacy, before surgery. Postoperatively, geriatric comanagement allows for early, aggressive management of delirium, pain, mobility, nutrition, and multimorbidity, which may improve outcomes, particularly in high-risk individuals with cognitive impairment. Larger studies are needed to confirm these findings, although if our results bear out, it will indicate the 
importance of identifying preoperative cognitive impairment. Identification of these individuals, who traditionally have a much higher perioperative risk, will allow for targeted resource allocation and ideally risk mitigation using a geriatric comanagement model.

SLUMS is a brief, validated assessment tool that screens for MCI and dementia. ${ }^{19,31}$ SLUMS content emphasizes executive functioning and episodic memory, allowing earlier detection of subtle deficits associated with MCI than the Mini-Mental State Examination. ${ }^{32}$ SLUMS takes approximately 10 minutes to administer, making it a viable option in a busy preoperative setting, and has demonstrated diagnostic utility for MCI and dementia similar to that of longer screening tests, such as the Montreal Cognitive Assessment. ${ }^{33}$ Despite the benefits of SLUMS, there is a paucity of data examining whether a person's performance is linked to health outcomes. A single study found that lower SLUMS scores were associated with greater risk of institutionalization and death in an ambulatory veteran population, but no studies have specifically examined the role of SLUMS in preoperative screening. ${ }^{34}$ Our data suggest that preoperative SLUMS scores may have limited capacity to predict POD (highest c-statistic on ROC $=0.54$ ).

This study must be considered in light of its limitations. It was a small, exploratory study and may have been underpowered to detect meaningful differences in outcomes of populations with and without cognitive impairment. The imbalance in the sample sizes of participants with and without cognitive impairment (two-thirds of the study group having cognitive impairment) further limits our ability to detect differences. In addition, we had a highly educated population, and more than half of the study population was referred from neurosurgery services. This may limit the generalizability of the data.

In summary, cognitive impairment is logically associated with adverse health outcomes for older adults in the perioperative period. Cognitive disorders affect one's ability to properly manage medications, care for wounds, and engage with ancillary providers such as physical therapists. Identification of higher risk individuals, particularly when considering comanagement with a geriatrics team, allows for appropriate resource use.

More research is needed to develop valid measures of cognitive impairment that are sensitive enough to detect meaningful cognitive impairment but brief enough to allow convenient deployment in a busy preoperative clinical setting. Moreover, specific cognitive domains, such as executive functioning, may have stronger predictive capacity for postoperative outcomes. ${ }^{35,36}$ Understanding the relationship between these domains and risk of delirium would allow for better identification of the highest risk individuals and for targeted optimization and resource allocation. Such strategies will help surgeons and patients better understand perioperative risks and benefits, encouraging effective shared decision-making models.

\section{ACKNOWLEDGMENTS}

The authors would like to acknowledge Heather Whitson, $\mathrm{MD}$, for her assistance in reviewing this manuscript.

Financial Disclosure: Eugene Stead Award, Department of Internal Medicine, Duke University Health
Systems; The Brookdale Foundation, The Hartford Foundation, Health Resources and Services Administration, and NIH/NHLBI HL109971.

Conflict of Interest: Dr. McDonald and Dr. Heflin receive grant support from the Department of Veterans Affairs Office of Academic Affiliation for a Specialty Care Education Center of Excellence to develop the VA POSH program. Dr. Heflin is an author for Up to Date, Inc.

Author Contributions: Dr. Zietlow had full access to all of the data in the study and takes responsibility for the integrity of the data and the accuracy of the data analysis. Zietlow: Study concept and design, data analysis, data interpretation, manuscript drafting, critical revision of manuscript. McDonald, Sloane, Browndyke, Lagoo-Deenadayalan: Study design, data analysis, data interpretation, manuscript drafting, critical revision of manuscript. Heflin: Study concept and design; supervision; data acquisition, analysis, and interpretation; manuscript drafting; critical revision of manuscript.

Sponsor's Role: Sponsors provided funding for salary support, statistical analysis, and manuscript editing services.

\section{REFERENCES}

1. Oresanya LB, Lyons WL, Finlayson E. Preoperative assessment of the older patient: A narrative review. JAMA 2014;311:2110-2120.

2. Ortman JM, Velkoff VA, Hogan H. An Aging Nation: The Older Population in the United States (Report P25-1140) [on-line]. Available at https:// www.census.gov/library/publications/2014/demo/p25-1140.html Accessed June 18, 2017.

3. American Society of Anesthesiologists Physical Status Classification System [on-line]. Available at https://www.asahq.org/resources/clinical-information/ asa-physical-status-classification-system Accessed June 18, 2017.

4. Tran Ba Loc P, du Montcel ST, Duron JJ et al. Elderly POSSUM, a dedicated score for prediction of mortality and morbidity after major colorectal surgery in older patients. Br J Surg 2010;97:396-403.

5. Chow WB, Rosenthal RA, Merkow RP et al. Optimal preoperative assessment of the geriatric surgical patient: A best practices guideline from the American College of Surgeons National Surgical Quality Improvement Program and the American Geriatrics Society. J Am Coll Surg 2012;215:453466.

6. Cohen RR, Lagoo-Deenadayalan SA, Heflin MT et al. Exploring predictors of complication in older surgical patients: A deficit accumulation index and the Braden Scale. J Am Geriatr Soc 2012;60:1609-1615.

7. Partridge JS, Harari D, Martin FC et al. The impact of pre-operative comprehensive geriatric assessment on postoperative outcomes in older patients undergoing scheduled surgery: A systematic review. Anaesthesia 2014;69:8-16.

8. McDonald SR, Heflin MT, Whitson HE et al. Association of integrated care coordination with postsurgical outcomes in high-risk older adults: The Perioperative Optimization of Senior Health (POSH) initiative. JAMA Surg 2017; https://doi.org/10.1001/jamasurg.2018.5513.

9. Kim S, Brooks AK, Groban L. Preoperative assessment of the older surgical patient: Honing in on geriatric syndromes. Clin Interv Aging 2014;10:13-27.

10. Ansaloni L, Catena F, Chattat R et al. Risk factors and incidence of postoperative delirium in elderly patients after elective and emergency surgery. Br J Surg 2010;97:273-280.

11. Raats JW, Steunenberg SL, de Lange DC et al. Risk factors of post-operative delirium after elective vascular surgery in the elderly: A systematic review. Int J Surg 2016;35:1-6.

12. Saczynski JS, Marcantonio ER, Quach L et al. Cognitive trajectories after postoperative delirium. N Engl J Med 2012;367:30-39.

13. Devore EE, Fong TG, Marcantonio ER et al. Prediction of long-term cognitive decline following postoperative delirium in older adults. J Gerontol A Biol Sci Med Sci 2017;72:1697-1702.

14. Aykut K, Albayrak G, Guzeloglu M et al. Preoperative mild cognitive dysfunction predicts pulmonary complications after coronary artery bypass graft surgery. J Cardiothorac Vasc Anesth 2013;27:1267-1270.

15. Messerotti Benvenuti S, Patron E, Zanatta P et al. Preexisting cognitive status is associated with reduced behavioral functional capacity in patients 
3 months after cardiac surgery: An extension study. Gen Hosp Psychiatry 2014:36:368-374.

16. Daigle CR, Schauer PR, Heinberg LJ. Bariatric surgery in the cognitively impaired. Surg Obes Relat Dis 2015;11:711-714.

17. Miller MD, Paradis CF, Houck PR et al. Rating chronic medical illness burden in geropsychiatric practice and research: Application of the Cumulative Illness Rating Scale. Psychiatry Res 1992;41:237-248.

18. Mundi S, Chaudhry H, Bhandari M. Systematic review on the inclusion of patients with cognitive impairment in hip fracture trials: A missed opportunity? Can J Surg 2014;57:E141-E145.

19. Tariq SH, Tumosa N, Chibnall JT et al. Comparison of the Saint Louis University Mental Status Examination and the Mini-Mental State Examination for detecting dementia and mild neurocognitive disorder-a pilot study. Am J Geriatr Psychiatry 2006;14:900-910.

20. Sindi S, Mangialasche F, Kivipelto M. Advances in the prevention of Alzheimer's disease. F1000Prime Rep 2015;7:50.

21. Salvi F, Miller MD, Grilli A et al. A manual of guidelines to score the modified Cumulative Illness Rating Scale and its validation in acute hospitalized elderly patients. J Am Geriatr Soc 2008;56:1926-1931.

22. Boustani M, Campbell N, Munger S et al. Impact of anticholinergics on the aging brain: A review and practical application. Aging Health 2008;4:311-320.

23. Harris PA, Taylor R, Thielke $\mathrm{R}$ et al. Research electronic data capture (REDCap) - a metadata-driven methodology and workflow process for providing translational research informatics support. J Biomed Inform 2009; 42:377-381.

24. Inouye SK, van Dyck CH, Alessi CA et al. Clarifying confusion: The confusion assessment method. A new method for detection of delirium. Ann Intern Med 1990;113:941-948.

25. Koskderelioglu A, Onder O, Gucuyener $\mathrm{M}$ et al. Screening for postoperative delirium in patients with acute hip fracture: Assessment of predictive factors. Geriatr Gerontol Int 2017;17:919-924.

26. Benedetti MG, Ginex V, Mariani E et al. Cognitive impairment is a negative short-term and long-term prognostic factor in elderly patients with hip fracture. Eur J Phys Rehabil Med 2015;51:815-823.

27. Kistler EA, Nicholas JA, Kates SL et al. Frailty and short-term outcomes in patients with hip fracture. Geriatr Orthop Surg Rehabil 2015;6:209-214.

28. Pogliacomi F, Schiavi P, Defilippo M et al. Total elbow arthroplasty following complex fractures of the distal humerus: results in patients over 65 years of age. Acta Biomed 2016;87:148-155.

29. Culley DJ, Flaherty D, Fahey MC et al. Poor performance on a preoperative cognitive screening test predicts postoperative complications in older orthopedic surgical patients. Anesthesiology 2017;127:765-774.
30. Jones RN, Marcantonio ER, Saczynski JS et al. Preoperative cognitive performance dominates risk for delirium among older adults. J Geriatr Psychiatry Neurol 2016;29:320-327.

31. Feliciano L, Horning SM, Klebe KJ et al. Utility of the SLUMS as a cognitive screening tool among a nonveteran sample of older adults. Am J Geriatr Psychiatry 2013;21:623-630.

32. Stewart S, O'Riley A, Edelstein B et al. A preliminary comparison of three cognitive screening instruments in long term care: The MMSE, SLUMS, and MoCA. Clin Gerontol 2012;35:57-75.

33. Cummings-Vaughn LA, Chavakula NN, Malmstrom TK et al. Veterans Affairs Saint Louis University Mental Status examination compared with the Montreal Cognitive Assessment and the Short Test of Mental Status. J Am Geriatr Soc 2014;62:1341-1346.

34. Cruz-Oliver DM, Malmstrom TK, Allen CM et al. The Veterans Affairs Saint Louis University Mental Status exam (SLUMS exam) and the MiniMental Status Exam as predictors of mortality and institutionalization. J Nutr Health Aging 2012;16:636-641.

35. Smith PJ, Attix DK, Weldon BC et al. Executive function and depression as independent risk factors for postoperative delirium. Anesthesiology 2009;110:781-787.

36. Rudolph JL, Jones RN, Grande LJ et al. Impaired executive function is associated with delirium after coronary artery bypass graft surgery. J Am Geriatr Soc 2006;54:937-941.

\section{SUPPORTING INFORMATION}

Additional Supporting Information may be found in the online version of this article:

Figure S1. Relationship between SLUMS score and rates of postoperative delirium

Please note: Wiley-Blackwell is not responsible for the content, accuracy, errors, or functionality of any supporting materials supplied by the authors. Any queries (other than missing material) should be directed to the corresponding author for the article. 